\title{
Reviewers for the 2019 IMIA Yearbook of Medical Informatics
}

\author{
Mervat Abdelhak, USA \\ Julian Alvarez, France \\ Robert Amland, USA \\ Elske Ammenwerth, Austria \\ Avalos Marta, France \\ Cheick Oumar Bagayoko, Mali \\ Panagiotis Bamidis, Greece \\ Melissa Baysari, Australia \\ Tim Benson, United Kingdom \\ Jiang Bian, USA \\ Georgeta Bordea, France \\ Sue Bowman, USA \\ Andrew Boyd, USA \\ Celia Boyer, Switzerland \\ David Buckeridge, Canada \\ Alex Bui, USA \\ John Carrino, USA \\ Pascal Charbonel, France \\ Jonathan H. Chen, USA \\ Rémy Choquet, France \\ Christopher G. Chute, USA \\ Kevin Bretonnel Cohen, USA \\ Carolina Gómez Conejo, Spain \\ Pascal Coorevits, Belgium \\ Theresa Cullen, USA \\ David Darmon, France \\ Hans Demski, Germany \\ Gayo Diallo, France \\ Brian Dixon, USA \\ Alevina Dubovitskaya, Switzerland \\ Stephany Duda, USA \\ Martin Dugas, Germany \\ Margo Edmunds, USA
}

\author{
Frédéric Ehrler, Switzerland \\ Urs Eisenmann, Germany \\ Noémie Elhadad, USA \\ Peter Elkin, USA \\ Peter Embi, USA \\ William Erdley, USA \\ Susan Fenton, USA \\ Xosé M Fernández, France \\ Giacomo Fiumara, Italy \\ Jason Alan Fries, USA \\ Walter Gall, Austria \\ Thomas Ganslandt, Germany \\ Jennifer Garvin, USA \\ Andrew Georgiou, Australia \\ Guido Giunti, Spain \\ Clément Goehrs, France \\ Kenneth Goodman, USA \\ Maria Hägglund, Sweden \\ Thierry Hamon, France \\ Sébastien Harispe, France \\ Ralf Hofestaedt, Germany \\ Shannon Houser, USA \\ Lukas Huber, Austria \\ Josef Ingenerf, Germany \\ Trevor Jamieson, Canada \\ Igor Jurisica, Canada \\ Johanna Kaipio, Finland \\ Jayashree Kalpathy-Cramer, USA \\ David Kaufman, USA \\ Halil Kilicoglu, USA \\ Jeffrey Klann Jefrey, USA \\ Sebastian Köhler, Germany \\ Dimitrios Kokkinakis, Sweden
}

Mayank Kumar, India

Craig Kuziemsky, Canada

Antoine Lamer, France

Paul Landais, France

Thomas A. Lasko, USA

Nathan Lea, United Kingdom

Thierry Lecroq, France

Nelly Leon-Chisen, USA

Siaw-Teng Liaw, Australia

Frank Lin, Australia

Christian Lovis, Switzerland

Gang Luo, USA

Nadia Madaoui, France

Bradley A. Malin, USA

Romaric Marilly, France

Luis Marco-Ruiz, Norway

Mar Marcos, Spain

Santiago Martinez, Norway

Catalina Costa Martínez, Austria

Mark Merolli, Australia

Anne Moen, Norway

Hans Moen, Finland

Pattanasak Mongkolwat, Thailand

Shawn Murphy, USA

Radha Nagarajan, USA

Aurélie Névéol, France

Zahra Niazkhani, Iran

Stacy O'Connor, USA

Casey Overby Taylor, USA

Bunyamin Ozaydin, USA

Philip R. 0. Payne, USA

Niels Peek, United Kingdom

Mor Peleg, srrael
David Pieczkiewicz, USA
Andrea Pinna, France
Habibollah Pirnejad, Iran
Marie-Cecile Ploy, France
Morgan Price, Canada
Laritza Rodriguez, USA
Lipika Samal, USA
Neil Sarkar, USA
Matthieu Schuers, France
Marco Schweitzer, Austria
Chaitanya Shivade, USA
Hardeep Singh, USA
Berglind Smaradottir, Norway
Nathalie Sout, France
William Speier, USA
Olivier Steichen, France
Felix Sukums, Tanzania
Hugues Talbot, France
Xavier Tannier, France
Cui Tao, USA
Frantz Thiessard, France
Ye Tian, USA
Umit Topaloglu, USA
Pierre-Yves Vandenbussche, The Netherlands
Sumithra Velupillai, Sweden
Karin Verspoor, Australia
Amy Wang, USA
Chunhua Weng, USA
Alfred Winter, Germany
Klaus-Hendrik Wolf, Germany
Hua Xu, USA
Pierre Zweigenbaum, France
a 